\title{
"Comment peut-on être cochon ? : darwinisme et conscience chez Darrieussecq
}

\section{Simon Kemp}

Number 115, Winter 2020

Précisions sur les sciences dans l'oeuvre de Marie Darrieussecq

URI: https://id.erudit.org/iderudit/1067881ar

DOI: https://doi.org/10.7202/1067881ar

See table of contents

Publisher(s)

Department of French, Dalhousie University

ISSN

0711-8813 (print)

2562-8704 (digital)

Explore this journal

\section{Cite this article}

Kemp, S. (2020). « Comment peut-on être cochon ? » : darwinisme et conscience chez Darrieussecq. Dalhousie French Studies, (115), 21-28.

https://doi.org/10.7202/1067881ar

\section{Article abstract}

The confluence of cognitive science and Darwinian theory has produced a wealth of fascinating research in recent decades, often enthusiastically embraced by the humanities, and given rise to the new disciplines of evolutionary psychology and neurophenomenology. Darrieussecq's interest in human and non-human cognition makes her work a site of exploration for several issues related to these fields of research. Her characters speculate on the inner lives and perceptual worlds of dogs, cats and insects. The narrative focalization of her story will leap unexpectedly from a human consciousness into that of a sea-lion or a basking shark. And, most famously, in Darrieussecq's debut novel we follow the narrator's subjectivity as she metamorphoses between human and pig form and states undecidably in-between, during which process, we gradually realize, it is not only her physical form that is shifting but her mentality as well. This article examines how Darrieussecq's exploration of animal consciousness and its relation to the human not only serves as a metaphor for intersubjectivity and the unknowable mind of the other, but also offers a meditation on the nature of humanity and of its place within an evolutionary spectrum of differently adapted minds.
This document is protected by copyright law. Use of the services of Érudit (including reproduction) is subject to its terms and conditions, which can be viewed online.

https://apropos.erudit.org/en/users/policy-on-use/ 


\title{
«Comment peut-on être cochon ?": darwinisme et conscience chez Darrieussecq
}

\author{
Simon Kemp
}

$\mathcal{L}$ es liens de plus en plus étroits entre les sciences cognitives et la théorie darwinienne ont permis de faire des découvertes importantes au cours des dernières décennies en ce qui concerne l'esprit humain et sa place dans l'immense diversité des êtres vivants de notre planète. Souvent adoptées par les sciences humaines avec enthousiasme, ces théories ont engendré par leur confluence de nouvelles disciplines comme la psychologie évolutionniste et la neuro-phénoménologie. Comme le note Shirley Jordan (55), Darrieussecq a toujours été fascinée par les potentialités littéraires offertes par les sciences, passant de la physique subatomique de Naissance des fantômes (1999) au clonage de Notre vie dans les forêts (2017). L'intérêt de Darrieussecq pour la cognition humaine et animale fait de son œuvre un site d'exploration de plusieurs questions liées à ces domaines de recherche. La conscience non humaine est un thème central dans son œuvre depuis le début de sa carrière d'écrivaine. Ses personnages s'interrogent sur la vie intérieure de plusieurs espèces animales, comme par exemple l'otarie, ou encore le chat, l'oiseau, le poisson et la mouche dans Naissance des fantômes, ou bien le chien, à nouveau le chat, et le chimpanzé dans Bref séjour chez les vivants (2001). Le lecteur accède directement au cerveau d'un requin pèlerin dans Le Mal de mer (1998), et partage la perspective d'un être parfois femme, parfois truie, parfois mélange incertain des deux, dans son exploration la plus célèbre de la bête humaine, Truismes (1996). Dans cet article, il s'agit d'examiner la représentation de la conscience non-humaine dans les textes de Darrieussecq. L'analyse vise à démontrer que l'exploration de la conscience animale et humaine chez Darrieussecq ne sert pas seulement de métaphore de l'intersubjectivité et de la difficulté à comprendre la conscience de l'autre, mais offre aussi une méditation sur ce qu'est l'humanité et sur sa place dans un spectre darwinien de cerveaux différemment adaptés. Malgré un intérêt véritable de la part de l'écrivaine pour la conscience des animaux, il s'avérera néanmoins que la principale préoccupation de sa fiction reste à tout moment la subjectivité humaine, et que la représentation de la conscience animale sert avant tout à mettre cette dernière en valeur. Il s'agira de montrer comment Darrieussecq évoque la nature de la conscience animale, ayant recours à des moyens allant de la neuroanatomie et la théorie darwinienne à l'empathie imaginative et la métaphore poétique, et comment les similarités et les contrastes qu'elle révèle entre cette nature et l'esprit humain éclairent les caractéristiques de notre propre subjectivité.

\section{La conscience des animaux}

L'étude de la conscience non humaine est plus souvent présente dans les premiers livres de Darrieussecq. Dans ses romans plus récents, son intérêt pour la psychologie subsiste : le cerveau humain, l'aspect physique de l'émotion et de la sensation, et la conscience logée dans le cerveau et dans le corps restent des thèmes majeurs, mais elle se tourne plus rarement vers le monde animalier pour les illustrer. C'est dans ses quatre premiers romans - Truismes, Naissance des fantômes, Le Mal de mer et Bref séjour - qu'elle privilégie le cerveau animal, et tout particulièrement dans le premier et le dernier livre de ce quatuor de textes. Commençons entre ces deux pôles de la grande étude du devenir-animal de Truismes et de celle de l'être humain de Bref séjour par les épisodes relativement mineurs de Naissance des fantômes et du Mal de mer qui ont trait à la conscience non humaine. Ces épisodes concernent tous deux la conscience d'un animal marin : l'otarie dans Naissance des fantômes et le requin pèlerin dans Le Mal de mer. 
Dans le passage suivant, tiré de Naissance des fantômes, la narratrice frappe le mâle dominant d'un groupe d'otaries d'une pierre, puis imagine la cognition qui sous-tend sa réaction tardive :

[D]e toutes mes forces j'ai lancé un caillou, pile entre les deux yeux que l'abruti a ouverts un peu plus grand, l'information a cheminé à travers ses synapses bardées de lard, deux neurones ont réussi à se détacher de leur graisse pour inaugurer leur roulement à billes et j'ai vu la grosse masse sans plus de queue ni tête se déplacer comme un asticot vers moi. (65)

Dans le fantasme de la narratrice, le cerveau de l'otarie est imaginé au niveau neurologique, perçu sous l'angle de l'activité électrochimique des cellules de sa cervelle. Elle ne parle pas de l'expérience phénoménologique de l'animal. Il n'est même pas certain qu'il y ait une subjectivité qui accompagne la réponse tropiste au stimulus. Stimulé par une pierre, l'animal répond par un mouvement et, selon la narratrice du roman, il n'est pas clair si ce mouvement est le résultat d'une sensation de douleur suivie par une décision consciente ou d'un simple réflexe mécanique.

Dans Le Mal de mer, nous retrouvons cette même perspective scientifique et neurologique en ce qui concerne le requin pèlerin. Le narrateur nous informe

[qu']une étude récente du Musée de la Mer a prouvé qu'il ne se déplaçait pas au gré des courants, comme son apparente passivité et son faible développement neuronal pouvaient le laisser supposer, mais selon des circuits stratégiques. (128)

Au contraire de l'épisode de l'otarie, le narrateur impersonnel et hétérodiégétique du roman permet ici non seulement d'offrir la perspective neuronale de ce qui se passe dans la tête du requin, mais aussi de traverser de l'autre côté du "problème difficile de la conscience », comme l'a appelé le philosophe David Chalmers, pour évoquer les expériences qualitatives créées par cette activité cérébrale :

La fatigue a remplacé la faim. Le vide ouvert sous ses fanons usés semble s'être clos peu à peu, la mer ne le traverse plus, elle rencontre un obstacle au fond du ventre, un calme. Les muscles n'ont plus à se mouvoir pour échapper à cette faim, le corps se laisse porter comme une bouée. (Le Mal de mer 125-26)

Avec le requin pèlerin, Darrieussecq a choisi de pénétrer un esprit très différent de celui de l'être humain, un esprit vraiment étranger. Ce 'faible développement neuronal' ne permet peut-être même pas une subjectivité proprement dite ; l'animal vit sans conscience de soi avec des pensées, des sensations et des perceptions étrangères à celles que nous reconnaîtrions.

Le monde phénoménologique du requin nous montre comment Darrieussecq se sert de la cognition animale pour méditer sur les façons différentes de percevoir le réel. Dans Naissance des fantômes, cette différence devient une métaphore du fossé intraversable entre les perspectives de deux êtres humains, fussent-ils mari et femme : la narratrice se demande à la fin du roman «si mon mari (si les chats, les oiseaux, les poissons et les mouches aux yeux à facettes) sentait et voyait tout de même ce que moi je sentais et voyais » (158). La question posée sur la perception animale et humaine est en effet une façon pour la narratrice de reconnaître qu'elle n'a jamais vraiment connu ni son mari, ni la perspective de celui-ci sur leur couple.

Darrieussecq revient à ce thème de perception comparative de façon plus littérale dans Bref séjour, un roman qui, selon Jordan, " aborde la question de ce qu'il veut dire, au cours de ce nouveau siècle, d'être humain » $(66)^{1}$. Nore, la plus jeune des trois sœurs dont nous

1 « A novel approaching the question of what, in this new century, it will mean to be human ». 
suivons l'histoire, se met à imaginer comment la plage où elle vient de se promener ferait impression sur l'esprit de son chat :

Je me réveille chat, et je n'ai plus qu'à descendre quémander ma pâtée, après avoir rêvé ma vie d'humain... le courant bleu au large sur la... verte... il verrait quoi, une étendue hostile et pas d'ombre. Le sable, mou sous les pattes. Les algues à odeur de poisson. (206)

La perception est donc une construction mentale fabriquée à partir des données sensorielles et, Darrieussecq nous suggère, c'est une construction contingente, organisée différemment selon la personne ou l'animal qui perçoit. Comme dans la philosophie de Heidegger, le monde est vu non tel qu'il est, mais selon les projets de celui qui regarde. Ce qui nous est utile (les choses «à portée de main », zuhanden, dans la terminologie de Heidegger) est privilégié, ainsi que les choses potentiellement nocives, alors que ce qui est sans rapport avec notre vie s'efface (Heidegger 101-08). Un chat accorde la priorité à des choses différentes de celles favorisées par la priorité humaine, et le rivage est donc un monde différent au travers des yeux d'un félin de ce qu'il serait à nos yeux. Nous pouvons également observer un parallèle avec les recherches sur la perception dans les sciences cognitives contemporaines. Selon le neuroscientifique V. S. Ramachandran, notre perception est loin d'être un enregistrement objectif de la réalité que nous avons devant nous ; nous devrions plutôt parler d'un cerveau qui choisit l'hallucination qui s'accorde le mieux avec les données reçues (229). Ce «best-fit hallucination», une construction virtuelle créée à partir de la réalité extérieure, mais nullement synonyme de cette réalité, est un modèle de la perception souvent utilisé dans l'écriture de Darrieussecq.

\section{Le cerveau hybride et la continuité mentale}

Ce ne sont pas seulement les différences entre le cerveau humain et animal qui attirent l'attention de l'écrivaine. Dans son étude de la représentation des animaux de Darrieussecq, Anne Simon fait référence à une "mixité charnelle et mentale avec les bêtes » (81) que partagent les personnages humains de ses romans :

Tout se passe comme si bêtes et humains, appartenant à l'ensemble plus vaste du vivant, possédant des origines cellulaires et/ou marines communes, étaient envisagés dans un continuum dont on a repéré les dangers, mais qui autorise en retour, via les images du faisceau et du réseau, un accès démultiplié au monde et à soi. (81)

Ce domaine du vivant sert de contexte pour la psychologie humaine que Darrieussecq explore, et ce sont souvent des liens de similarité qu'elle remarque entre l'univers mental de l'humain et de l'animal. Dans Bref séjour, l'écart entre les animaux et l'enfant humain ne semble pas particulièrement important. Anne, nouveau-née, a « le poil mouillé d'un petit animal » (22), par exemple. Quand la toute petite Nore regarde un chimpanzé au zoo, il n'est pas évident pour sa sœur aînée de décider lequel des deux serait le plus doué intellectuellement :

[L]e petit chimpanzé exactement de la taille de Nore qui la regardait, la regardait [...] et elle, un toddler encore, gros paquet de couches sur pattes, petit bonnet blanc sur les boucles châtain nous cessons vite d'être blondes, Pierre l'était resté jusqu'au bout - et qui regardait qui on se demandait, ça a des yeux humains pathétiques, et des mains humaines, et ça s'assoit ça s'asseyait mieux que Nore, ça saisissait entre pouce et index mieux que Nore, à se demander si au jeu du 
triangle dans le triangle elle aurait été, question méninges, la mieux lotie... $(202)^{2}$

Adolescente, Nore est « troublée » par le tressautement de paupières d'un chat dormant, et qui implique que nous ne sommes pas la seule espèce à rêver pendant le sommeil (36). Même dans la perception, elle voit des liens, à la différence de la narratrice de Naissance des fantômes. Nore note que le cochon et la pieuvre ont les yeux les plus similaires à ceux de l'homme, et considère la possibilité que nous partagions une même vision du monde (205). Bien sûr, la possession d'un système visuel comparable n'implique pas que les données visuelles seront interprétées de la même manière par la pieuvre, le cochon et l'être humain, qui habitent chacun un monde très différent, avec des besoins et des priorités incommensurables. Même si le champ de vision de la pieuvre ressemble à celui de l'humain, les systèmes de perception dans le cerveau de cette créature pourraient être d'un tout autre ordre : il est difficile d'imaginer ce qui serait « à portée de main » du point de vue d'une pieuvre.

Néanmoins, ce jeu de similarités dans Bref séjour brouille les frontières entre le cerveau humain et le cerveau animal. Le roman de Darrieussecq qui va le plus loin à cet égard est, bien sûr, son premier, Truismes. Dans ce roman, les métamorphoses de femme en truie de la narratrice, et, dans une moindre mesure, celles de son amant loup-garou, offrent au lecteur un témoignage fictif du vécu d'être humain, d'être animal, et, pour la plupart du roman, d'être hybride indécidable situé quelque part entre les deux. Dans une étude du roman dans le contexte de la littérature de métamorphose, Naama Harel observe qu'il y a dans cette dernière une convention de ce qu'elle appelle « the psycho-physical split » (398), c'est-à-dire une division entre le corps du personnage - qui se transforme en animal - et l'esprit, qui n'est pas affecté par le changement. Mis à part quelques évolutions dans les préférences et habitudes du personnage, comme le nouveau faible de Gregor Samsa pour la nourriture pourrie après sa transformation en insecte dans La Métamorphose de Kafka, la littérature de métamorphose nous offre l'histoire d'un esprit humain dans un corps animal, et pour Harel, le roman de Darrieussecq fait lui aussi partie de cette tendance. Certes, la narratrice retient des traits mentaux caractéristiques de l'humain dans son corps de truie. Les autres cochons se méfient d'elle : «ils sentent bien que ça continue à penser comme les hommes là-dedans » (141). L'auteur a même dit de son personnage : "Pour moi, à la fin elle est beaucoup plus humaine qu'au début. Quelle que soit son apparence » (Darrieussecq et Gaudet 111). Dans sa nouvelle vie de monstre et de paria, la narratrice apprend à réévaluer les idées reçues selon lesquelles elle vivait son ancienne vie, et à reconnaitre la société misogyne qui a abusé d'elle. Elle acquiert aussi le pouvoir de s'exprimer, pouvoir qui se manifeste dans l'acte de narration lui-même. Pourtant, Harel a assurément tort d'affirmer que le roman présente une métamorphose purement corporelle. La pensée humaine perçue par les autres cochons dans la porcherie est retenue par la narratrice seulement en partie et avec grande difficulté, comme on le remarque clairement lorsqu'elle essaie de se concentrer sur son évasion :

J'ai essayé de faire tourner ce fichu verrou avec la patte, avec le groin, mais je n'y arrivais pas, mon corps ne comprenait pas pourquoi il devait s'acharner sur

2 Il est également question d'un chimpanzé à l'esprit presque humain dans la nouvelle 'Connaissance des singes' (2005), reprise dans la collection Zoo (2006). Dans cette histoire fantastique, la narratrice s'occupe d'un chimpanzé qui sait parler : 'Il maniait les mots avec précaution, articulant du bout des lèvres, la bouche en cul de poule. D'un être humain on eût dit qu'il était maniéré' (47). Au cours de l'histoire, le chimpanzé parle de moins en moins et commence à reprendre le comportement d'un singe normal: 'J'écrivais, il mangeait, il reprenait du poil de la bête. Il passait le balai dans toute la maison, il faisait des grimaces dans le miroir, il rigolait en poursuivant les oiseaux. Nous n'avions pas grand'chose à nous dire, mais le silence ne nous pesait plus' (53). La relation entre la narratrice et le singe rest néanmoins une relation d'amitié et de compréhension mutuelle. 
cette pièce d'acier, mon corps se mouvait sans conviction alors que tous mes neurones s'épuisaient à garder cette idée en tête, le verrou, le verrou, c'était épuisant de lutter ainsi contre soi-même. (145)

Ailleurs dans le roman, elle oublie des éléments de la vie quotidienne, comme le fait que la messe soit célébrée le dimanche. "Je devenais un peu bête » (74), s'excuse-t-elle, et il semble que la narratrice ne remarque pas le double-sens de ses paroles. Lorsqu'elle découvre qu'elle ne sait plus lire, loin d'être frustrée, elle se plaît à manger les livres ; au même moment elle se met à manger des cadavres et à dormir dans ses propres excréments. L'odorat remplace la vision comme sens principal. L'épisode le plus clair - et le plus troublant - d'une mentalité métamorphosée arrive quand la narratrice trouve sur le trottoir un bébé dans un siège-auto qu'une mère s'apprête à mettre dans la voiture, vraisemblablement pour fuir un conjoint abusif. La vérité de cette situation frappe la narratrice « comme un éclair de compréhension » (84), rétrospectivement après le départ de la femme. Pendant la rencontre elle-même, en revanche, peu de choses dans la cognition de la narratrice évoquent le monde humain. Elle fouille du nez les affaires de la femme sur le trottoir, elle pousse le bébé d'un coup de groin et ne sait plus si les bruits qu'elle provoque sont des rires ou des hurlements de terreur. (Pour le lecteur, il est clair que ce ne sont pas des rires.) Pire encore, il semble dans le moment qu'il existe un risque bien réel qu'elle tue et mange l'enfant : «Il me semble, comment dire, que ça m'aurait été facile de le manger, de planter mes dents dans cette chair bien rose » (84).

Truismes, bien sûr, est avant tout un roman satirique sur le sexisme et la brutalité d'une société patriarcale, plutôt qu'une analyse de la pensée non-humaine ${ }^{3}$. L'animal en lequel la narratrice se métamorphose est non seulement l'espèce sus domesticus, mais aussi la "grosse truie » de l'insulte misogyne. Cependant, un roman comme Truismes ne se limite pas à une seule signification, et ce texte, accompagné par les trois romans qui l'ont suivi, fait partie d'une méditation sur la place de l'humain et la nature de la conscience humaine. «[Les animaux] nous obligent à une métaphysique instantanée : qu'est-ce qui nous sépare d'eux ? Qu'est-ce qui nous fait humain ? ", demande l'écrivaine lors d'un entretien (Darrieussecq, Miller et Holmes s. p.). Dans son œuvre, Darrieussecq affirme tout d'abord que la conscience non-humaine existe ; elle rejette la conception cartésienne de l'animal comme automate inconscient, et rejette également la tradition philosophique qui ferait du langage une condition préalable à l'accès à la conscience. En outre, en situant les esprits humains et animaux sur un continuum mental, elle représente l'humain comme rien de plus qu'un « mammifère pensant » (170), comme se définit la narratrice du roman Le Pays. Cela la situe en opposition avec la conception religieuse de l'humanité, pour laquelle l'humain est le seul être doué d'une âme, et la place à l'opposé des doctrines laïques « exceptionnalistes », comme l'existentialisme de Sartre, qui réserve l'être-pour-soi pour l'humain, et abandonne les animaux à l'être-en-soi, au même ordre d'existence que les plantes et les pierres. Le «mammifère pensant» de Darrieussecq établit un lien entre sa représentation de l'humain et les théories de la psychologie évolutionniste, qui cherchent des parallèles entre le comportement humain et animal pour démontrer l'origine adaptive de nos traits psychologiques. En même temps, les théories de la cognition animale qui sont fondées sur une base darwinienne trouvent souvent leur écho dans la représentation de Darrieussecq. Daniel C. Dennett propose dans Kinds of Minds : Towards an Understanding of Consciousness (1996) une continuité mentale des reactions automatiques des organismes les plus simples jusqu'à la vie intérieure complexe de l'humain, dans laquelle on ne voit pas de frontière définitive qui démarquerait l'automatisme de la conscience proprement

3 Voir à ce sujet, par exemple : Lucile Desblache, « La truie, féminité et animalité » (143-52); Jeannette Gaudet, "Dishing the Dirt : Metamorphosis in Marie Darrieussecq's Truismes »; Julie Rodgers, "Body Politics in Truismes : the Tyranny of Slenderness »; ou Sonja Stojanovic, "'J'ai mangé, j'ai mangé' [I ate and ate] : Accumulation and Excess in Marie Darrieussecq's Truismes ». 
dite. Bien que la capacité linguistique du cerveau humain confère, selon lui, un caractère réflexif à notre conscience qui la distingue de celle des autres mammifères, la conscience non-linguistique des animaux reste néanmoins une conscience, remplie de pensées, d'émotions et de sensations muettes, qui pourraient être conçues et décrites par l'esprit humain par moyen d'empathie ${ }^{4}$.

L'emphase sur terrain commun entre l'univers mental de l'animal et celui de l'humain permet à Darrieussecq de mettre en valeur certains aspects de la psychologie humaine parfois négligés par la représentation littéraire. Dans l'écriture de Darrieussecq, la pensée non-linguistique est au premier plan. Les sentiments, les émotions, les sensations et l'imagerie semblent le plus souvent mener une vie autonome dans l'esprit du personnage, sans lien à la parole parlée ou pensée. Une citation de Naissance des fantômes illustre bien cette tendance :

Mes muscles se tendaient, mon ventre mollissait. Mes nerfs subissaient une traction interne. C'était le vide qui me vidait de l'intérieur, comme un pauvre poulet, de ma chair et de ma pensée. Je sentais sous mon sternum des écoulements, mais l'air autour de moi était parfaitement immobile, indifférent à la ponction. [...] Une atmosphère solide pesait sur mes joues, sur mes bras, sur mes jambes ; une cendre en pétrification, de plus en plus épaisse, me moulait. (84)

La dépression et l'inertie qu'éprouve la protagoniste après la disparition de son mari sont communiquées au lecteur par des images qui font penser aux tropismes de Sarraute : un liquide qui coule, une carapace qui emprisonne. Racontée rétrospectivement, mais vécue sur le moment sans recours à la parole interne ou externe, l'expérience mentale et émotionnelle de la protagoniste n'est pas loin de celles vécues par les animaux représentés ailleurs dans l'œuvre de Darrieussecq. Ces expériences animales, comme la joie d'un chien, la peur d'un cochon, ou le malaise d'un requin, qui ont lieu dans un cerveau dépourvu de fonctions cognitives supérieures, peuvent être décrites dans un discours similaire à celui de la narratrice de Naissance des fantômes, créant ainsi un rapprochement entre l'univers mental humain et celui de l'animal. De plus, à la différence des tropismes de Sarraute, qui sont des métaphores pour représenter un événement purement abstrait dans l'esprit, dont l'image souvent biologique souligne l'aspect réflexif ou instinctif, la biologie dans les images de Darrieussecq n'est nullement métaphorique. Les sensations décrites sont réellement perçues par le personnage comme ayant lieu dans le corps, sous la peau, dans la cage thoracique, dans les bras et les jambes, et c'est à travers ces sensations physiques que le personnage ainsi que le lecteur arrivent à comprendre son état d'esprit. Le cerveau dans l'écriture de Darrieussecq est un cerveau incarné, et la vie mentale est vécue dans et par le corps physique. C'est un état d'esprit ancré dans le corporel, tout comme la peur d'un cochon, qui se manifeste en « une odeur de victime » (Truismes 154) que dégage le corps, comme la perception d'un chat focalisée par des pattes sur le sable mou, ou comme un requin troublé par un calme au fond de son ventre dans les eaux trop peu profondes près du rivage.

La position philosophique de Darrieussecq dans ces textes est loin du célèbre « devenir-animal » de Deleuze et Guattari (284-380), qui parle d'une perte d'individualité humaine dans la masse indifférenciée de l'animalité. Il s'agit donc plutôt du contraire de

4 Dennett l'exprime ainsi : 'The idea that a dog's "thought" might be inexpressible (in human language) for the simple reason that expression in a human language cuts too fine is often ignored, along with its corollary : the idea that we may nevertheless exhaustively describe what we can't express, leaving no mysterious residue at all’ (42). (« On ignore souvent l'idée que la « pensée » d'un chien pourrait être inexprimable (dans le langage humain) pour la raison simple que l'expression dans le langage humain hache la chose trop menu, et on ignore aussi son corollaire : l'idée qu'on peut néanmoins décrire exhaustivement ce que nous ne pouvons pas exprimer, ne laissant aucun résidu mystérieux »). 
l'expérience de la narratrice de Truismes, qui se libère de son conformisme humain en se transformant en animal. Néanmoins, Darrieussecq affirme qu'elle trouve plusieurs parallèles entre sa pensée et celle de Deleuze et Guattari, bien qu'elle n'ait pas lu leur théorie du devenir-animal au moment d'écrire Truismes. Elle déclare dans Rapport de police «ni Derrida ni Foucault ni Barthes n'avaient théorisé si bien et si à l'avance ce qu'allait être mon imaginaire » (152), et cite les concepts deleuziens suivants comme liés à ses propres préoccupations créatives : « le devenir-animal, la déterritorialisation, le corps sans organes, les plis et les rhizomes » (152). Néanmoins, les parallèles avec la théorie de la conscience du philosophe américain, Thomas Nagel, sont plus évidents. Son essai majeur des années 70, «Quel effet cela fait-il d'être une chauve-souris? " (« What is it like to be a bat ?») fonde sa définition de la conscience sur la présence d'un vécu subjectif qui échappe aux tentatives de la neurophysiologie ou d'autres disciplines scientifiques de décrire cette conscience. Darrieussecq explore la neurophysiologie, comme on l'a vu dans l'exemple des synapses de l'otarie, ainsi que le vécu, comme dans l'exemple du requin pèlerin. Sa fiction démontre une fascination pour la relation entre ces deux phénomènes, et par la manière dont les cerveaux avec leurs différentes adaptations darwiniennes peuvent engendrer des subjectivités variées entre espèces, y compris l'espèce humaine.

\section{Conclusion}

De l'otarie au requin pèlerin, du chat au chimpanzé, nous avons vu comment la représentation du comportement extérieur et de la subjectivité intérieure des animaux fait partie de la méditation de Marie Darrieussecq sur la nature de la conscience et la place de l'humanité dans le monde des êtres vivants. Nous avons vu aussi comme l'écart entre l'humain et les autres espèces animales est réduit par l'hybride mi-animal mi-humain de Truismes, par la similitude entre singe et enfant dans Bref séjour, et, partout dans l'œuvre de Darrieussecq, par la mise en valeur dans la représentation de l'esprit humain de la pensée non-linguistique, de l'émotion muette, et de la sensation physique qui nous lient toujours à l'univers mental des animaux. Certes, l'écrivain ne projette pas d'effacer les différences énormes et évidentes qui nous séparent: la raison, la parole, et la réflexivité (ou la conscience de soi) restent hors de la portée de tout cerveau qui n'est pas un cerveau humain. Ce qui sous-tend toute l'écriture de Darrieussecq est la conviction que la conscience est un phénomène étendu, qui n'est pas exclusif à l'humanité, et que nous partageons avec toute une gamme d'autres animaux. Avec le chien sur la plage qui cligne des yeux pour éviter de rencontrer le regard de Nore, dans le chat qu'elle regarde rêver, et dans la femme d'un autre texte et d'un autre monde qui redécouvre sa liberté, son individualité et sa voix sous la forme d'une truie, Darrieussecq nous montre l'héritage commun de tous ces cousins éloignés sur leurs différentes branches de l'arbre évolutionnaire. À travers la comparaison, nous percevons plus clairement ce qu'est l'esprit humain.

Somerville College, Oxford

\section{OUVRAGES CITÉS}

Chalmers, David. "Facing up to the Problem of Consciousness ». Journal of Consciousness Studies 2 (1995) : 200-19. Imprimé.

Darrieussecq, Marie. Truismes. Paris : P.O.L, 1996. Imprimé.

---. Naissance des fantômes. Paris : P.O.L, 1998. Imprimé.

---. Le Mal de mer. Paris : P.O.L, 1999. Imprimé.

---. Bref séjour chez les vivants. Paris : P.O.L, 2001. Imprimé.

---. Le Pays. Paris : P.O.L, 2005. Imprimé.

---. Zoo. Paris : P.O.L, 2006. Imprimé.

---. « Être libéré de soi ». Le Magazine littéraire (Mars 2008) : 58. Imprimé. 
---. Rapport de police-Accusations de plagiat et autres modes de surveillance de la fiction. Paris : P.O.L, 2010. Imprimé.

---. Notre vie dans les forêts. Paris : P.O.L, 2017. Imprimé.

Darrieussecq, Marie et Jeannette Gaudet. «'Des livres sur la liberté' : conversation avec Marie Darrieussecq ». Dalhousie French Studies 59 (2002) : 108-18. Imprimé.

Darrieussecq, Marie, Becky Miller et Martha Holmes. «Entretien avec Marie Darrieussecq ». University of Arizona (Décembre 2001). Site Marie Darrieusecq. http://www.mariedarrieussecq.com/entretiens. (Dernière consultation 1 août 2019). Web.

Deleuze, Gilles et Félix Guattari. «Devenir-intense, devenir-animal, devenirimperceptible ». Capitalisme et Schizophrénie 2 : Mille Plateaux. Paris : Minuit, 1980. 284-380. Imprimé.

Dennett, Daniel C. Kinds of Minds: Towards an Understanding of Consciousness. New York : Perseus, 1996.

Desblache, Lucile. "La truie, féminité et animalité ». Bestiaire du roman contemporain d'expression française. 143-52. Clermont-Ferrand : P U Blaise Pascal, 2002.

Gaudet, Jeannette. « Dishing the Dirt: Metamorphosis in Marie Darrieussecq's Truismes ». Women in French Studies 9 (2001) : 181-92.

Harel, Naama. " Challenging the Species Barrier in Metamorphosis Literature: The Case of Marie Darrieussecq's Pig Tales ». Comparative Critical Studies 2 (2005) : 397-409. Imprimé.

Heidegger, Martin. Being and Time. Trad. John Macquarrie et Edward Robinson. Oxford : Blackwell, 1962. Imprimé.

Jordan, Shirley. "'Un grand coup de pied dans le château de cubes': Formal Experimentation in Marie Darrieussecq's Bref Séjour chez les vivants ». Modern Language Review 100.1 (2005) : 51-67. Imprimé.

Nagel, Thomas. «What is it like to be a bat? » Philosophical Review 83.4 (1974) : 43550. Imprimé.

Ramachandran, V. S. The Tell-Tale Brain: Unlocking the Mystery of Human Nature. London : Heinemann, 2011. Imprimé.

Rodgers, Julie. "Body Politics in Truismes: the Tyranny of Slenderness ». Dalhousie French Studies 98 (2012) : 29-37. Imprimé

Simon, Anne. « Marie Darrieussecq ou la plongée dans les 'mondes animaux' ». Dalhousie French Studies 98 (2012) : 77-87. Imprimé.

Stojanovic, Sonja. "'J'ai mangé, j'ai mangé' [I ate and ate] : Accumulation and Excess in Marie Darrieussecq's Truismes ». Starvation, Food Obsession and Identity: Eating Disorders in Contemporary Women's Writing. Dir. Petra M. Bagley, Francesca Calamita et Kathryn Robson. Oxford : Peter Lang, 2018. 167-86. Imprimé. 